\title{
DENGUE 2 (NEW GUINEA C STRAIN) VIRUS INFECTION INDUCES APOPTOSIS
}

\author{
Norazizah Shafee and Sazaly AbuBakar* \\ Department of Medical Microbiology, Foculty of Medicine, Universiti Malaya, 50603 Kuala Lumpur, Maloysia
}

\begin{abstract}
The ability of dengue 2 virus infection to induce apoptosis was investigated. Dengue 2 virus-infected African green monkey kidney cells (Vero), showed the presence of about $2,7,22$ and 30 percent apoptotic cells on day 3, 5, 8, and 11 postinfection, respectively. Increased number of apoptotic cells corresponded with increasing number of dengue 2 antigen positive cells. Induction of internucleosomal DNA fragmentation, characteristic of apoptotic cells, was noted also in dengue 2 virus-infected mosquito cell $(\mathrm{C} 6 / 36)$ cultures. These results suggested that dengue 2 virus infection in vitro stimulated apoptotic cellular responses. (JUMMEC 1997 2(1): 19 - 21)
\end{abstract}

KEYWORDS: Apoptosis, C6/36, dengue, Vero

\section{Introduction}

Apoptosis is characterized by a series of co-ordinately regulated cellular events which culminate in cell death. The process involved distinct biochemical and cellular events which differentiate it from cell death by necrosis (1). Since it's initial description, the importance of apoptosis in various cellular functions has been unravelled. More recently, apoptosis is noted to play a significant role in the demise of the CD4+ cells in HIV infection (2). A number of other viruses including adenovirus, sindbis virus, influenza virus, bovine herpesvirus, and lymphocytic choriomeningitis virus have also been associated with induction of apoptosis (reviewed in 3). On the other hand, there are viruses which are known to encode proteins which inhibit apoptosis. These include the T antigen, E6, LMPI, CrmA and p35 protein of SV40, human papilloma virus, Epstein-Barr virus, cowpox virus, and baculovirus, respectively (3). The ability to cause or inhibit apoptosis has been associated to various forms of manifestations of the virus infection.

Dengue virus, a mosquito borne RNA virus is known to cause infection which affects the host intravascular functions (4). The mechanisms whereby the virus causes them, however, are still unknown. In the present study, we investigated the potential induction of apoptosis by dengue 2 virus infection in vitro.

\section{Materials and Methods}

\section{Cells and virus preparation}

Vero cells used in this study were obtained from the American Type Culture Collection (ATCC, Rockville, MD, USA). Cells were cultured on glass coverslips in
RPMI 1640 medium (Gibco BRL, Grand Island, NY,USA) supplemented with $10 \%$ fetal calf serum (PAA Laboratories, Linz, Austria). Cells were infected only when they were about $80-90 \%$ confluent. In a typical experiment, infection was done to give an estimated multiplicity of infection (MOI) of about $0.1-0.5$ plaque forming unit (PFU) per cell. Dengue 2 New Guinea $C$ (NGC) strain virus propagated using the $\mathrm{C} 6 / 36$ cells was used entirely in this study.

\section{Detection of apoptotic cells}

Apoptotic cells were detected using the Apoptosis Detection System (Promega, Madison,WI, USA). Briefly, dengue 2 virus-infected or mock-treated Vero cells were fixed in 10\% paraformaldehyde, washed with phosphate buffered solution (PBS) and permeabilized using $0.5 \%$ Triton-X 100. The slides were then rinsed in PBS and finally with distilled water. All procedures were done on ice. Slides were air-dried and kept at $4^{\circ} \mathrm{C}$ until needed for staining.

Staining using terminal deoxynucleotidyl transferase (TdT)-mediated dUTP nick-end labelling (TUNEL) was performed using fluorescein-12-dUTP following the protocols provided by the kit's manufacturer (Promega, Madison, WI, USA). Samples were viewed using a Zeiss Axiolab fluorescence microscope (Zeiss, Germany) using standard fluorescein excitation and emission filters. Using this labelling procedure, the apoptotic cells' DNA were labelled with fluorescein-12-dUTP, thus, appeared green.

-Corresponding address:

Sazaly AbuBakar, Ph.D.

Department of Medical Microbiology. Faculty of Medicine, Universiti Malaya,

50603 Kuala Lumpur, Malaysia.

Email: sazaly@medicine.med.um.edu.my 
DNA isolation. Dengue 2 virus-infected or mock-treated C $6 / 36$ cells were lysed in lysis solution consisting of $I \%$ $\mathrm{N}$-lauryl-sarcosine, $0.2 \%$ sodium deoxycholate, and proteinase $\mathrm{K}(\mathrm{I} \mathrm{mg} / \mathrm{ml})$ in L-Buffer $(0.01 \mathrm{MTris}-\mathrm{HCl}, \mathrm{pH} 7.6$; $0.02 \mathrm{M} \mathrm{NaCl} ; 0.1 \mathrm{M}$ EDTA). Equal amount of DNA then was electrophoresed in a $1.8 \%$ agarose gel prepared in $0.5 \times$ TAE (0.02 M Tris-base; $0.01 \mathrm{M}$ sodium acetate, 0.5 $\mathrm{mM}$ EDTA) buffer. Following electrophoresis, agarose gels were stained with ethidium bromide and photographed under ultraviolet light illumination at $302 \mathrm{~nm}$ wavelength.

\section{Detection of dengue virus antigens}

The presence of dengue virus antigens in the infected cells was determined using pooled mouse anti dengue 2 virus hyperimmune sera and TRITC-conjugated antimouse IgG (Sigma Chemical Company, St. Louis, MO, USA). All dengue antigen positive cells stained red when viewed using the TRITC filters under fluorescent microscopy.

\section{Results and Discussion}

Dengue 2 virus-infected Vero cells showed characteristic cellular morphological changes after about 5 days post-infection (PI). Increasing number of cells were noted to 'float-off' from the monolayer with increasing

Table I. Percentage of apoptotic cells in dengue 2 virus-infected Vero cell cultures. The number of cells stained positive for dengue virus antigens, apoptotic cells, and the total number of cells per microscopic field were counted. The mean of at least 10 microscopic fields per datum was calculated and the percentages were determined by dividing the means (mean number of apoptotic cells or mean number of dengue antigen positive cells per field/mean number of cells per field $x$ 100).

\begin{tabular}{lcccccc}
\hline & \multicolumn{6}{c}{ Days post-infection (PI) } \\
& 0 & 1 & 3 & 5 & 8 & 11 \\
\hline Mean number of cells & 11 & 25 & 54 & 125 & 165 & 133 \\
Percent infected $( \pm$ S.D) & 0 & 0 & $2(2)$ & $28(6)$ & $32(10)$ & $97(3)$ \\
Percent apoptotic ( \pm S.D) & 0 & 0 & I(I) & $7(2)$ & $22(5)$ & $31(9)$ \\
\hline
\end{tabular}

number of days $\mathrm{PI}$. As expected no dengue virus antigen positive cells or apoptotic cells were noted in the mock-treated samples prepared until day II PI (data not shown). The number of dengue antigen positive cells and apoptotic cells as determined by immunofluorescent staining and TUNEL respectively, increased significantly $(\mathrm{P}<0.001$, ANOVA) beginning on day $3 \mathrm{PI}$, si- multaneously with increasing number of cells (Table I). By day $8 \mathrm{PI}$, the mean number of cells per microscopic field reached plateau. Many of the apoptotic cells were seen to 'float-off' from the cell monolayers by day $8 \mathrm{PI}$, thus, reducing the total number of cells counted. Whereas, the number of dengue positive cells increased dramatically reaching almost $97 \%$ of the remaining cells counted by day II PI. It was noted that the apoptotic cells stained positive for dengue virus antigens (Figure I). A very good correlation ( $r>0.75$, Pearson's) between the number of apoptotic cells and the number of dengue virus antigen positive cells was observed. These results suggested that dengue 2 NGC strain virus infection could induce apoptotic responses.

Induction of apoptosis was noted also in C6/36 mosquito cells infected with dengue 2 virus. In these cells, internucleosomal fragmentation of the cellular DNA into multiples of about 180-200 bp, characteristics of apoptotic cells' DNA, was observed on day $8 \mathrm{PI}$ (Figure 2 ), suggesting that dengue virus 2 infection induces apoptosis potentially in all susceptible cells. Further investigations are on-going in our laboratory to confirm this suggestion.

Induction of apoptosis by dengue virus I was previously reported (5). In this study, the mouse neuroblastoma cells were infected with dengue I virus inoculum

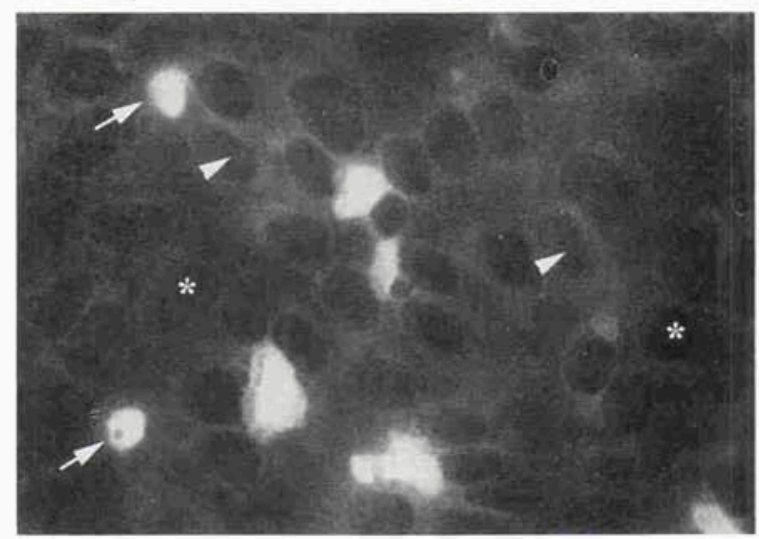

Figure I. Photomicrograph of apoptotic cells in dengue 2 virus infected Vero cell cultures. Vero cells cultured on glass coverslips were treated with dengue 2 virus inoculum to give an estimated $\mathrm{MOI}$ of about 0.1 to 0.5 PFU/cell. On day $5 \mathrm{PI}$ cells were fixed and doubly stained for the presence of dengue virus antigens and apoptotic cells. The image shown was digitized using a Kodak Professional RFS2035 Plus Film Scanner and labelling was done using the Adobe Photoshop 3.0. The photograph was printed using the Kodak XLS8600 PS Printer. Arrow heads ( ) indicate cells showing the presence of only dengue virus antigens; arrows $\Leftrightarrow$ indicate the apoptotic cells expressing dengue virus antigens, and asteriks $\left({ }^{*}\right)$ indicate non-apoptotic cells showing no virus antigens. 


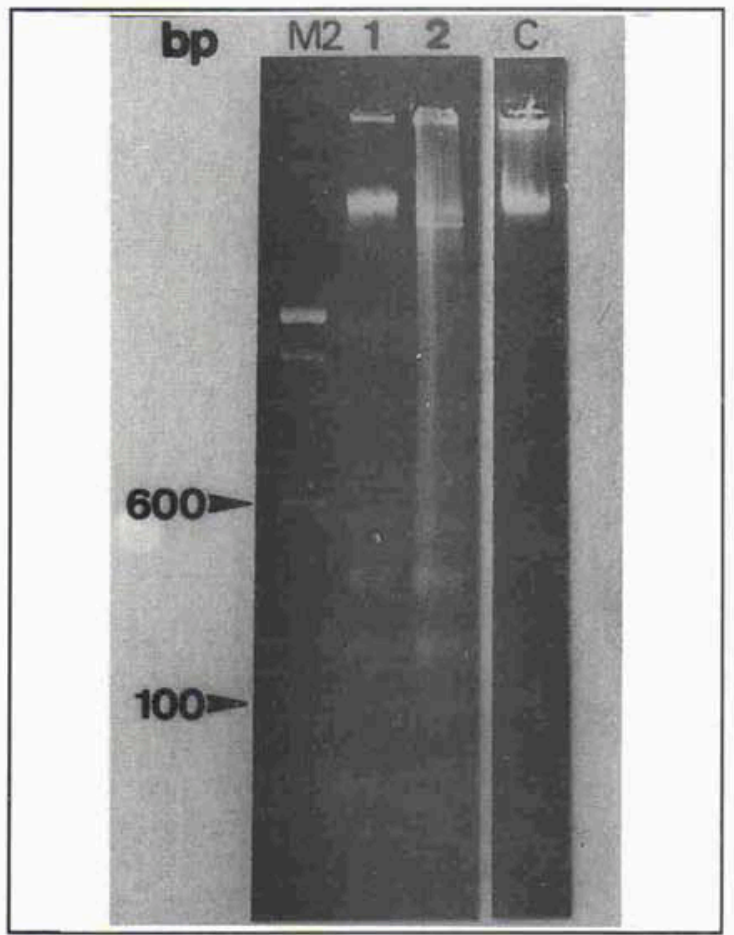

Figure 2. Agarose gel electrophoresis of DNA prepared from dengue 2 virus infected and mock-treated C6/36 cells. Monolayer of C $6 / 36$ cells $(80-90 \%$ confluent) were infected with dengue 2 virus to give an estimated $\mathrm{MOI}$ of about 0.1-0.5 PFU/cell. At selected intervals post-infection, DNA was extracted and electrophoresed following the procedures as described in the Materials and Methods. 100 bp marker (M2); mock-treated (C); day 8 PI (lane I); and day I5 PI (lane 2 ). The mock treated cells' DNA was prepared on day $18 \mathrm{PI}$. at about 400 PFU/cell. Apoptosis was noted by $25 \mathrm{hr}$ PI. In contrast, in the present investigation the Vero cells were infected at a very low MOI of 0.1-0.5 PFU/ cell. This low MOI which probably simulates closely the in vivo infection, resulted in induction of detectable level of apoptosis only on day $3 \mathrm{PI}$. Nevertheless, these findings support the notion that induction of apoptosis by dengue virus infection could play an important role in the overall pathogenesis of dengue virus infection. Our laboratory is presently investigating to determine if clinical isolates of dengue viruses could also induce apoptosis. We hope to be able to demonstrate that dengue virus-induced apoptosis do occur in vivo.

\section{Acknowledgments}

This investigation was funded by the Ministry of Science, Technology and Environment R\&D IRPA Grants \# 03-07-04-503 and \# 06-02-03-0303.

\section{References}

I. Duvall E andWyllie AH. Death and the cell. Immunology Today 1986; 7:115-9.

2. Groux H, Torpier G. Monte D, Mouton Y, Capron A and Ameisen JC. Activation-induced death by apoptosis in $C D 4+T$ cells from human immunodeficiency virus-infected asymptomatic individuals. J Exp Med 1992; 175:331. 40.

3. Shen $Y$ and ShenkTE. Viruses and apoptosis. Curr Opin Genet Dev 1995; 5: 105-11.

4. Bhamarapravati N. Hemostatic defects in dengue hemorrhagic fever. Rev Infect Diseases 1989; 44 (Supl) : S826-9.

5. Despres P, Flamand M, Ceccaldi PI and DeubelV. Human isolates of dengue type I virus induce apoptosis in mouse neuroblastoma cells. JVirol 1996; 70: 4090-6. 